\title{
Macroscopic Quantum Phase Interference in Antiferromagnetic Particles
}

\author{
Yi-Hang Nie ${ }^{1,2}$,Yan-Hong Jin ${ }^{1,5}$, \\ J.-Q.Liang ${ }^{1,3}$, H.J.W.Müller-Kirsten ${ }^{3}$,D.K.Park ${ }^{3,4}$, F.-C.Pu ${ }^{5,6}$ \\ ${ }^{1}$ Institute of Theoretical Physics and Department of Physics,Shanxi University, \\ Taiyuan,Shanxi 030006, People's Republic of China \\ ${ }^{2}$ Department of physics, Yanbei Normal Institute, Datong Shanxi 037000, \\ People's Republic of China \\ ${ }^{3}$ Department of Physics, University of Kaiserslautern D-67653 Kaiserslautern,Germany \\ ${ }^{4}$ Department of Physics, Kyungnam University, Masan, 631-701, Korea \\ ${ }^{5}$ Institute of Physics and Center for Condensed Matter Physics, \\ Chinese Academy of Sciences, Beijing 100080,People's Republic of China, \\ 6 Department of Physics, Guangzhou Normal College, Guangzhou 510400, \\ People's Republic of China
}

20 Dec. 1999

\begin{abstract}
The tunnel splitting in biaxial antiferromagnetic particles is studied with a magnetic field applied along the hard anisotropy axis. We observe the oscillation of tunnel splitting as a function of the magnetic field due to the quantum phase interference of two tunneling paths of opposite windings. The oscillation is similar to the recent experimental result with $\mathrm{Fe}_{8}$ molecular clusters.
\end{abstract}

The macroscopic quantum phenomenon of magnetic particles at low temperature has attracted considerable attention both theoretically and experimentally in recent years [1-3]. The magnetization vector in solids is traditionally viewed as a classical variable. The quantum transition of the magnetization vector $\mathbf{M}$ between different easy directions in a single domain ferromagnetic (FM) grain, in particular the coherent tunneling between two degenerate orientations of the magnetization called the macroscopic quantum 
coherence (MQC) [?], has been studied extensively for its exotic characters far from that of classical system. Quenching of MQC for half-integer spin is a fascinating effect [?, [0, (7, 8] and can be used to test the macroscopic quantum tunneling experimentally. The quenching of MQC in spin particles is analysed with the help of the phase interference of spin coherent state-paths which possess a phase with an obvious geometric meaning [5]. Although the quenching of MQC has been interpreted as Kramers' degeneracy, the effect of geometric phase interference is far richer than that. By investigating the quantum tunneling in biaxial ferromagnetic particles with a magnetic field applied along the hard axis Garg [9] found a new quenching of tunneling splitting which is not related to Kramers' degeneracy since the external field breaks the time reversal symmetry. The Zeeman energy of the biaxial spin particle in the external magnetic field results in additional topological phases of the tunnel paths which lead to the quantum phase interference. The tunneling splitting therefore oscillates with respect to the magnetic field.

According to a recent report [10] the oscillation of tunneling splitting was observed experimentally in molecular clusters $\mathrm{Fe}_{8}$ which at low temperature behavior like a nanomagnet, namely, a ferromegnetic particle. A more detailed analysis of quantum phase interference with instanton method in context of spin coherent-state-path-integrals has been given recently [11]. In the present letter we investigate the similar effect of quantum phase interference in antiferromagnetic (AFM) particles. Since the tunneling rate in AFM particles is much higher than that in the FM particles of the same volume [12], the AFM particles are expected to be a better candidate for the observation of MQP than the FM particles [12]. The AFM particle is usually described by the Néel vector of the two collinear sublattices whose magnetizations are coupled by strong exchange interaction. External magnetic field dose not play a role since the net magnetic moment vanishes for idealized sublattices. The quantum and classical transitions of the Néel vector in antiferromagnet have been well studied [13] in terms of the idealized sublattice model. The temperature dependence of quantum tunneling was also given for the same model [?] and the theoretical result agrees with the experimental observation [15]. A biaxial AFM particle with a small noncompensation of sublattices in the absence of an external magnetic field was studied in Ref. [16] where it was shown that the uncompensated magnetic moment leads to a modification of the oscillation frequency around the equilibrium orientations of the Néel vector. In the present letter we demonstrate that the uncompensated magnetic moment of a small biaxial AFM particle possesses a Zeeman energy in an external magnetic field applied along hard axis and thus a topological phase depending on the magnetic field is introduced similarly to the phase in ferromagnetic particles [9]. The quantum 
phase interference results in the oscillation of tunneling splitting as a function of magnetic field which may be regarded as a kind of the Aharonov-Bohm effect.

We consider in the following a biaxial AFM particle of two collinear FM sublattices with a small non-compensation. Assuming that the particle possesses a X easy axis and XY easy plane, and the magnetic field $h$ is applied along the hard axis ( $\mathrm{Z}$ axis), the Hamiltonian operator of the AFM particle has the form

$$
\hat{H}=\sum_{a=1,2}\left(k_{\perp} \hat{S}_{a}^{z 2}+k_{\|} \hat{S}_{a}^{y 2}-\gamma h \hat{S}_{a}^{z}\right)+J \hat{S}_{1} \cdot \hat{S}_{2}
$$

where $k_{\perp}, k_{\|}>0$ are the anisotropy constants, $J$ is the exchange constant, $\gamma$ is the gyromagnetic ratio,and the spin operators in two sublattices $\hat{S}_{1}$ and $\hat{S}_{2}$ obey the usual commutation relation $\left[\hat{S}_{a}^{i}, \hat{S}_{b}^{j}\right]=i \hbar \epsilon_{i j k} \delta_{a b} \hat{S}_{b}^{k}(i, j, k=x, y, z ; a, b=1,2)$. The matrix element of the evolution operator in spin coherent-state representation is

$$
\left\langle N_{f}\left|e^{-2 i \hat{H} T / \hbar}\right| N_{i}\right\rangle=\int\left[\prod_{k=1}^{M-1} d \mu\left(\mathrm{N}_{k}\right)\right]\left[\prod_{k=1}^{M}\left\langle\mathrm{~N}_{k}\left|e^{-i \epsilon \hat{H} / \hbar}\right| \mathrm{N}_{k-1}\right\rangle\right]
$$

Here we define $|\mathrm{N}\rangle=\left|\mathrm{n}_{1}\right\rangle\left|\mathrm{n}_{2}\right\rangle,\left|\mathrm{N}_{M}\right\rangle=\left|\mathrm{N}_{f}\right\rangle=\left|\mathrm{n}_{1, f}\right\rangle\left|\mathrm{n}_{2, f}\right\rangle,\left|\mathrm{N}_{o}\right\rangle=\left|\mathrm{N}_{i}\right\rangle=$ $\left|\mathrm{n}_{1, i}\right\rangle\left|\mathrm{n}_{2, i}\right\rangle, t_{f}-t_{i}=2 T$ and $\epsilon=2 T / M$. The spin coherent state is defined as

$$
\left|\mathrm{n}_{a}\right\rangle=e^{-i \theta_{a} \hat{C}}\left|S_{a}, S_{a}\right\rangle,(a=1,2)
$$

where $\mathrm{n}_{a}=\left(\sin \theta_{a} \cos \phi_{a}, \sin \theta_{a} \sin \phi_{a}, \cos \theta_{a}\right)$ is the unit vector, $\hat{\mathrm{C}}_{a}=\sin \phi_{a} \hat{S}_{a}^{x}-\cos \phi_{a} \hat{S}_{a}^{y}$ and $\left|S_{a}, S_{a}\right\rangle$ is the reference spin eigenstate. The measure is defined by

$$
d \mu\left(\mathrm{N}_{k}\right)=\prod_{a=1,2} \frac{2 S_{a}+1}{4 \pi} d \mathrm{n}_{a, k}, \quad d \mathrm{n}_{a, k}=\sin \theta_{a, k} d \theta_{a, k} d \phi_{a, k},
$$

In the large $S$ limit we obtain [17]

$$
\left\langle\mathrm{N}_{f}\left|e^{-2 i \hat{H} T / \hbar}\right| \mathrm{N}_{i}\right\rangle=e^{-i S_{0}\left(\phi_{f}-\phi_{i}\right)} \int \prod_{a=1,2} D\left[\theta_{a}\right] D\left[\phi_{a}\right] \exp \left(\frac{i}{\hbar} \int_{t_{i}}^{t_{f}} L d t\right)
$$

The Lagrangian is defined by $L=L_{0}+L_{1}$ with

$$
L_{0}=\sum_{a=1,2} S_{a} \dot{\phi}_{a} \cos \theta_{a}-J S_{1} S_{2}\left[\sin \theta_{1} \cos \theta_{2} \cos \left(\phi_{1}-\phi_{2}\right)+\cos \theta_{1} \cos \theta_{2}\right]
$$




$$
L_{1}=\sum_{a=1,2}\left(k_{\perp} S_{a}^{2} \cos ^{2} \theta+k_{\| \prime} S_{a}^{2} \sin ^{2} \theta_{a} \sin ^{2} \phi_{a}-\gamma h S_{a} \cos \theta_{a}\right)
$$

where $S_{0}=S_{1}+S_{2}$ is total spin of two sublattices. Since $S_{1}$ and $S_{2}$ is almost antiparallel, we may replace $\theta_{2}$ and $\phi_{2}$ by $\theta_{2}=\pi-\theta_{1}-\epsilon_{\theta}$ and $\phi_{2}=$ $\pi+\phi_{1}+\epsilon_{\phi}$, where $\epsilon_{\theta}$ and $\epsilon_{\phi}$ denote small fluctuations . Working out the fluctuation integrations over $\epsilon_{\theta}$ and $\epsilon_{\phi}$ the transition amplitude Eq.(5) reduces to

$$
\begin{aligned}
& \left\langle\mathrm{N}_{f}\left|e^{-2 i \hat{H} T / \hbar}\right| \mathrm{N}_{i}\right\rangle=e^{-i S_{0}\left(\phi_{f}-\phi_{i}\right)} \int \prod_{a=1,2} D[\theta] D[\phi] \exp \left(\frac{i}{\hbar} \int_{t_{i}}^{t_{f}} \bar{L} d t\right) \\
& \bar{L}=\Omega\left[\frac{m}{\gamma} \dot{\phi} \cos \theta+\frac{\chi_{\perp}}{\gamma} H \dot{\phi} \sin ^{2} \theta+\frac{\chi}{2 \gamma^{2}}\left(\dot{\theta}^{2}+\dot{\phi}^{2} \sin ^{2} \theta\right)\right]-V(\theta, \phi),
\end{aligned}
$$

where $V(\theta, \phi)=\left(\Omega K_{\perp} \cos ^{2} \theta+K_{\text {II }} \sin ^{2} \theta \sin ^{2} \phi-m h \cos \theta-\frac{\chi_{\perp}}{2} h^{2} \sin ^{2} \theta\right)$, and $\left(\theta_{1}, \phi_{1}\right)$ has been replaced by $(\theta, \phi) . m=\gamma \hbar\left(S_{1}-S_{2}\right) / \Omega$ with $\Omega$ being the volume of the AFM particle and $\chi_{\perp}=\frac{\gamma^{2}}{J} . K_{\perp}=2 k_{\perp} S^{2} / \Omega$ and $K_{\|}=2 k_{\|} S^{2} / \Omega$ (setting $S_{1}=S_{2}=S$ except in the term containing $S_{1}-S_{2}$ ) denote the transverse and the longitudinal anisotropy constants, respectively.

We assume a very strong transverse anisotropy, i.e. $K_{\perp} \gg K_{\| 1}$.In this case the Néel vector is forced to lie near the XY plane. Replacing $\theta$ by $\frac{\pi}{2}+$ $\eta$ where $\eta$ denotes the small fluctuation and carrying out the integral over $\eta$ we obtain

$$
\left\langle N_{f}\left|e^{-2 \hat{H} \beta / \hbar}\right| N_{i}\right\rangle=\int D[\phi] \exp \left(-\frac{1}{\hbar} \int_{\tau_{i}}^{\tau_{f}} L_{e f f} d \tau\right)
$$

where

$$
L_{e f f}=\frac{I}{2}\left(\frac{d \phi}{d \tau}\right)^{2}+i \Theta \frac{d \phi}{d \tau}+V(\phi)
$$

is the effective Euclidean Lagrangian. $\tau=i t$ and $\beta=i T . I=\Omega\left(I_{a}+I_{f}\right)$ where $I_{a}=m^{2} /\left(2 \gamma^{2} K_{\perp}\right)$ and $I_{f}=\chi_{\perp} / \gamma^{2}$ are the effective FM and AFM moments of inertia $\llbracket 18$, respectively. $V(\phi)=\Omega K_{\|} \sin ^{2} \phi$ is the effective potential and $\Theta=\hbar S_{0}-I \gamma h$. The second term in Eq.(11), i.e. $i \Theta \frac{d \phi}{d \tau}$ which is the total time derivative has no effect on the classical equation of motion, however it leads to a path dependent phase in Euclidean action. The classical equation of motion is seen to be 


$$
\frac{I}{2}\left(\frac{d \phi}{d \tau}\right)^{2}-V(\phi)=0
$$

$\phi=0$ and $\pi$ are two equilibrium orientation of the Néel vector. The Néel vector may transit by tunneling through potential barriers from one orientation $(\phi=0)$ to another $(\phi=\pi)$ along clockwise or anticlockwise paths. The instanton solutions of Eq.(12) are then obtained as

$$
\phi_{c}^{ \pm}(\tau)= \pm 2 \arctan \left(\mathrm{e}^{\omega_{0} \tau}\right)
$$

where $\omega_{0}=\sqrt{2 K_{\|} \Omega / I}$ is the small oscillation frequency of the Néel vector around its equilibrium orientation. $\phi_{c}^{-}(\tau)$ and $\phi_{c}^{+}(\tau)$ denote instanton solutions with clockwise and anticlockwise windings respectively. The Euclidean actions evaluated along the instanton trajectories are seen to be

$$
S_{E}^{ \pm}=\int L_{e f f} d \tau=2 I \omega_{0} \pm \Theta \pi
$$

The quantum phase interference of clockwise path "-" and anticlockwise path "+" is seen to be (see Fig.1)

$$
e^{-S_{E}^{+}}+e^{-S_{E}^{-}} \sim e^{-2 I \omega_{0} / \hbar} \cos (\Lambda \pi)
$$

where $\Lambda=\frac{\Theta}{\hbar}=S_{0}+\frac{h}{h_{c}}$ with $h_{c}=\frac{\hbar}{\gamma I}$. Since the potential $V(\phi)$ is periodic and can be regarded as a one-dimensional superlattice. Using the Bloch theory the low-lying energy spectrum could be determined as 19

$$
E_{0}=\varepsilon_{0}-2 \Delta \varepsilon_{0} \cos \pi(\Lambda+\xi)
$$

Where $\xi$ is Bloch wave vector which can be assumed to take either of the two values 0 and 1 [20]. $\Delta \varepsilon_{0}=\frac{2 \hbar \omega_{0}}{\pi} \mathrm{e}^{-2 I \omega_{0} / \hbar}$ is the usual overlap integral or simply the level shift induced by tunneling through any one of the barriers . Thus the tunneling splitting is seen to be

$$
\Delta \varepsilon=\left|2 \Delta \varepsilon_{0} \cos \pi(\Lambda+1)-2 \Delta \varepsilon_{0} \cos \pi \Lambda\right|=4 \Delta \varepsilon_{0}|\cos \pi \Lambda|
$$

which is a function of the external magnetic field like in the ferromagnetic particle case [9]1]. When $h=0$, Eq.(17) reduces to the previous result [16] where the tunneling splitting is quenched when $S_{0}=$ half-integer. With nonzero magnetic field the tunneling splitting would be quenched whenever $\Lambda=n+\frac{1}{2}$ or $h=\left(S_{0}-n-\frac{1}{2}\right) \hbar / I \gamma$ where $n$ is an integer. Fig.2 shows the oscillation of the tunneling splitting with respect to the external magnetic field. This quenching is due to the quantum phase interference of two tunneling paths of opposite windings. The period of oscillation is given by 


$$
\Delta h=\frac{\hbar}{I \gamma}
$$

We have demonstrated a macroscopic quantum interference effect in the tunneling of the magnetization of antiferromagnetic particles. Such particles open thus a new avenue to test macroscopic quantum interference effects. Experimental tests of our prediction could thus make an important contribution to our understanding of the transition region between the microscopic and the macroscopic world.

\section{Acknowledgements:}

This work was supported by the National Natural Science Foundation of China under Grant Nos. 1967701 and 19775033. J.-Q.L and D.K.P also acknowledge support by the Deutsche Forschungsgemeinschaft.

\section{References}

[1] Chudnovsky E M and Gunther L 1988 Phys. Rev. Lett. 60661

[2] Barbara B and Chudnovsky E M 1990 Phys. Lett. A 145205

[3] Gunther L and Barbara B (Eds) 1995 Procedings of the Meeting on Quantum Tunneling of Magnetization-QTM'94, Vol. 301 of NATO ASI series (Dordrecht: Kluwer)

[4] Garg A 1993 Phys. Rev. Lett. 714249

[5] Loss D DiVincenzo D Grinstein G 1992 Phys. Rev. Lett. 693232

[6] Chudnovsky E M and DiVincenzo D D 1993 Phys.Rev.B 4810548

[7] Prokofev N V and Stamp P C E 1993 J.Phys. Condens.Matter 5 L661

[8] Liang J -Q, Müller-Kirsten H J W and Zhou J -G 1997 Z. Physik. B 102525

[9] Garg A 1993 Europhys. Lett. 22209

[10] Wernsdorfer W and Sessoli R 1999 Science 284133

[11] Kou S -P, Liang J -Q, Zhang Y -B and Pu F -C 1999 Phys.Rev.B 59 11792

[12] J.M.Duan and A.Garg 1995 J. Phys.:Condense Matter 72171 
[13] Simanjuntak 1994 J.Phys.: Condens.Matter 62925

[14] Liang J -Q, Müller-Kirsten H J W, Nie Y-H, Pu F -C and Zhang Y -B 1998 Phys.Lett. A 248434

[15] Tejada J, Zhang X X 1994 J Phys.: Condes. Matter 6263

[16] Chudnovsky E M in:Gunth L and Barbara B (Eds) Quantum Tunneling of Magnetization QTM'94 pp 77-89

[17] Nie Y-H, ZhangY-B, Liang J -Q, Müller-Kirsten H J W and Pu F -C 1999 Physica B 27095

[18] Chudnovsky E M 1995 J Magn. Matter 140-144 1821

[19] Liang J -Q, Müller-Kirsten H J W, Zhou J -G, Zimmerschied F and Pu F -C 1997 Phys. Lett. B 393368

[20] Liang J -Q, ZhangY -B, Müller-Kirsten H J W, Zhou J -G, Zimmerschied F and Pu F -C 1998 Phys. Rev. B 57529

\section{Figure Caption:}

Fig.1: Quantum phase interference of two tunnel paths of opposite windings

Fig.2: Oscillation of tunneling splitting as a function of the external field with the solid line for $S_{0}=$ half-integer and the dotted line for $S_{0}=$ integer 


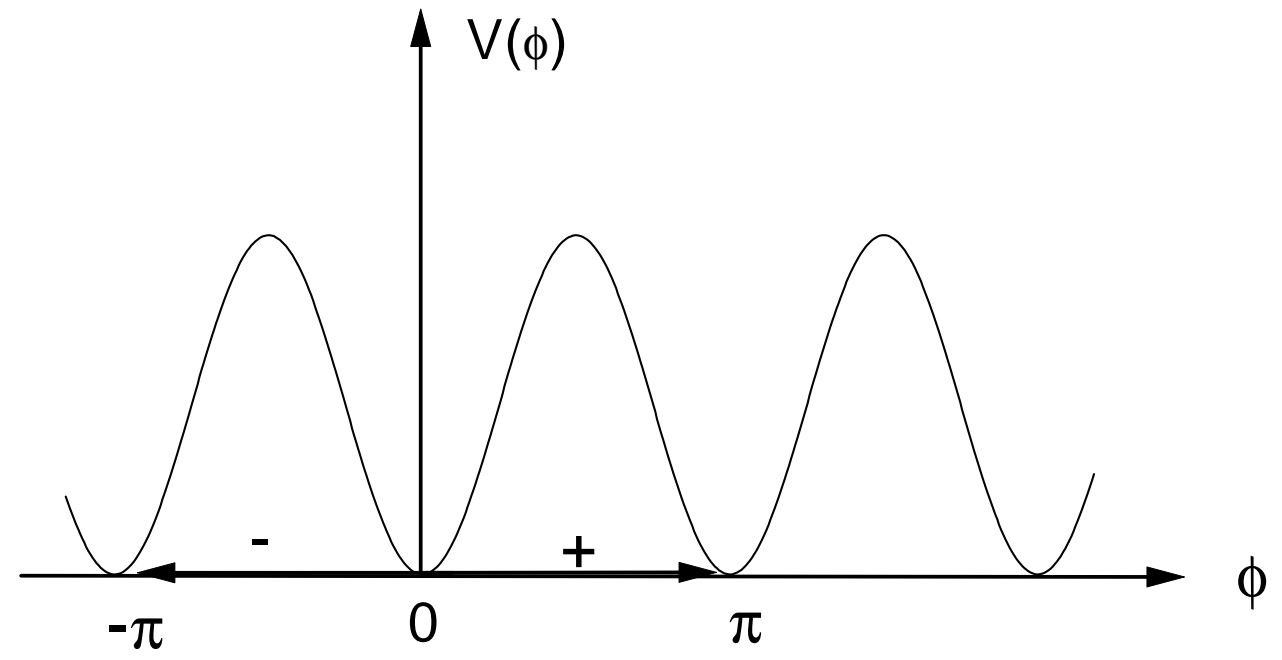

Fig.1 


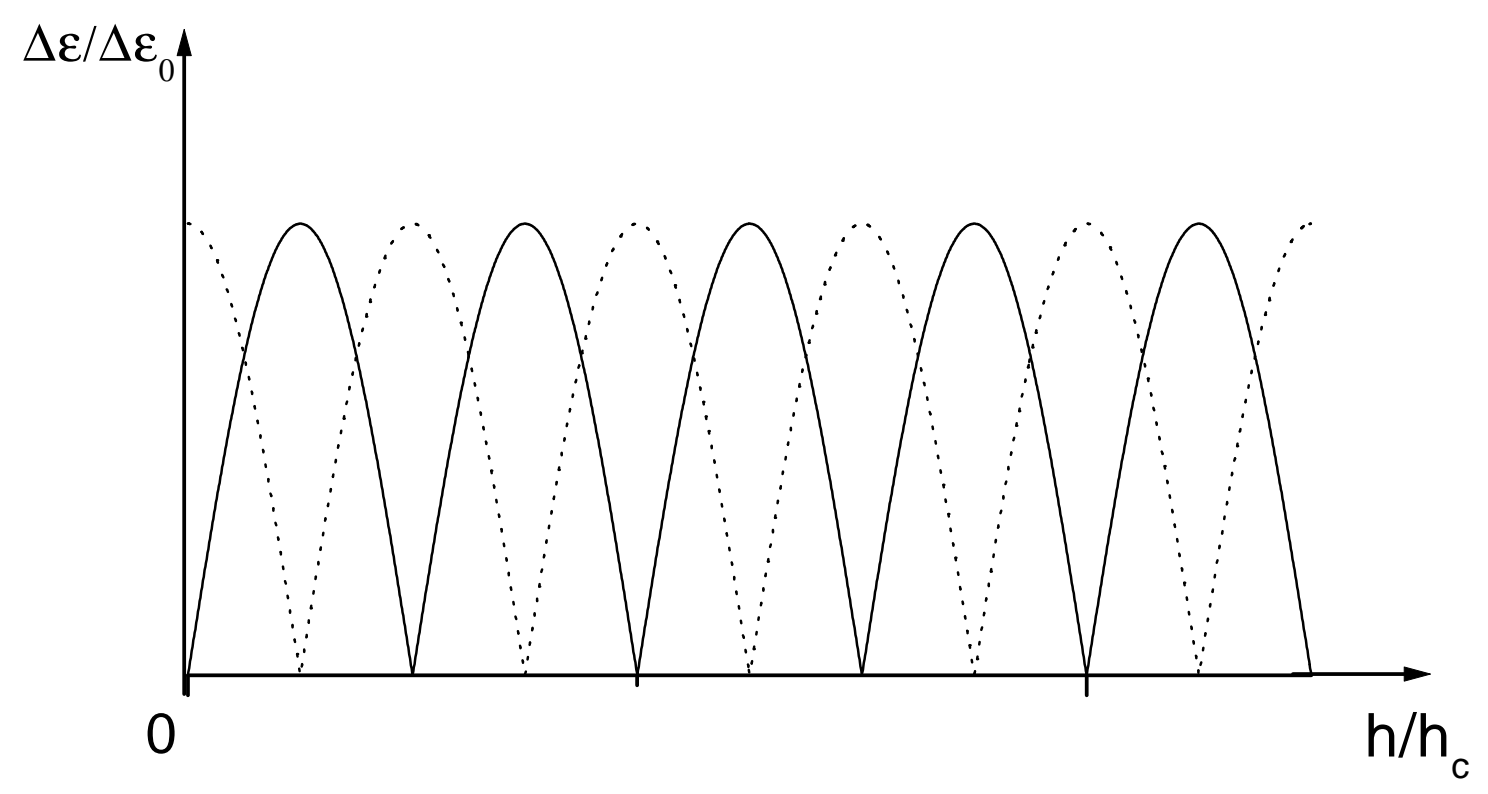

Fig. 2 